\title{
Emissões de $\mathrm{N}_{2} \mathrm{O}$ em Solos sob Cultivo de Cana-de-Açúcar no bioma Mata Atlântica: Efeito dos Sistemas de Colheita e da Adubação com Vinhaça
}

\author{
Lopes, I. M.; *Pinheiro, E. F. M.; Lima, E.; Ceddia, M. B.; Campos, D. V. B, \\ Alves, B. J. R.
}

Rev. Virtual Quim., 2017, 9 (5), 1930-1943. Data de publicação na Web: 31 de agosto de 2017 http://rvq.sbq.org.br

\section{Emissions of $\mathrm{N}_{2} \mathrm{O}$ in Soils under Sugarcane Cultivation in the Atlantic Forest biome: Effect of Harvesting Systems and Vinasse Fertilization}

\begin{abstract}
The conversion of areas under natural vegetation to agricultural areas is one of the major sources of greenhouse gas (GHG) emissions. The reason for the increase in GHG emissions is not only due to the loss of plant biomass but also to the greater decomposition of soil organic matter (SOM) due to agricultural management practices (fertilization, irrigation, residues burning, and mechanization). The objective of the study was to quantify nitrous oxide gas $\left(\mathrm{N}_{2} \mathrm{O}\right)$ flux in two traditional sites with sugarcane production, one with different straw management before harvest (burnt and unburnt) in Linhares (ES) and another one with different fertilization management (with and without vinasse application) in Campos dos Goytacazes (RJ), both sites inserted in the Brazilian Atlantic forest biome. The experiment carried out in Linhares, evaluated in October 2007 in an experimental area at the LAGRISA Plant had as treatments: sugarcane harvesting after straw burning (burnt) and cane harvesting without straw burning (unburnt) and surface straw distribution from soil. In the experiment conducted in Campos dos Goytacazes, evaluated in July 2008 treatments consisted of sugarcane without vinasse and with vinasse fertilization (single dose of $150 \mathrm{~m}^{3}$ ha ${ }^{-1}$ ). The $\mathrm{N}_{2} \mathrm{O}$ emission assessments were performed with static chambers, with six replicates per treatment, in both study areas. The $\mathrm{N}_{2} \mathrm{O}$ fluxes in Linhares site were not high, presenting a weekly average of ranging from 1.91 to $2.87 \mathrm{\mu g} \mathrm{m}^{-2} \mathrm{~h}^{-1}$ for the burnt and unburnt systems, respectively. The highest loss of $\mathrm{N}_{2} \mathrm{O}$ to the atmosphere occurred decreasingly from two days after the nitrogen fertilization together with the coverage of the residues in the raw cane system. In the Campos dos Goytacazes experimental site, the application of vinasse potentialized $\mathrm{N}_{2} \mathrm{O}$ flow, presenting high values $\left(195 \mu \mathrm{g} \mathrm{m} \mathrm{m}^{-2}\right)$ only in five hours after vinasse fertilization. The treatment without vinasse presented flow values close to zero $\left(0.4 \mu \mathrm{g} \mathrm{m}^{-2} \mathrm{~h}^{-1}\right)$, in the same gas sampling. After four days of vinasse application, there was no difference between treatments for $\mathrm{N}_{2} \mathrm{O}$ fluxes. The amount of organic matter easily assimilated to soil microorganisms, the increased in soil humidity due to vinasse addition and the precipitation occurring soon after vinasse fertilization were probably the most important variables in the control and magnitude of $\mathrm{N}_{2} \mathrm{O}$ emissions.
\end{abstract}

Keywords: Nitrous oxide; greenhouse gases flux; management of sugarcane straw; vinasse.

\section{Resumo}

A conversão de áreas sob vegetação natural para áreas agrícolas é uma das maiores fontes de emissão de gases de efeito estufa (GEE). O motivo do aumento da emissão de GEE não é somente devido a perda da biomassa vegetal decorrente da queima e preparo das áreas para o plantio, mas também pela maior decomposição da matéria orgânica do solo (MOS) decorrente das práticas de manejo agrícola (fertilização, irrigação, queima de resíduos culturais e mecanização). $\mathrm{O}$ objetivo deste estudo foi quantificar o fluxo do gás óxido nitroso $\left(\mathrm{N}_{2} \mathrm{O}\right)$ em duas áreas tradicionais de cultivo de cana-de-açúcar, uma com diferentes manejos da palhada perante a colheita (cana queimada e cana crua) em Linhares (ES) e outra área com diferentes manejos de adubação (com e sem aplicação de vinhaça) em Campos dos Goytacazes (RJ), ambas inseridas no bioma Mata Atlântica do Brasil. O experimento conduzido em Linhares, avaliado em outubro 2007 na área experimental na Usina LAGRISA teve como tratamentos: corte da cana após a queima da palhada (cana queimada) e corte da cana sem a queima da palhada (cana crua) e com distribuição da palha na superfície do solo. Já no experimento conduzido em Campos dos Goytacazes, avaliado em julho de 2008 os tratamentos consistiram de cana sem adubação de vinhaça e cana com adubação de vinhaça (dose única de $150 \mathrm{~m}^{3}$ ha ${ }^{-1}$ ). As avaliações das emissões de $\mathrm{N}_{2} \mathrm{O}$ foram realizadas com câmaras estáticas com seis repetições por tratamento em ambas as áreas de estudo. Os valores de fluxo de $\mathrm{N}_{2} \mathrm{O}$ em Linhares não foram elevados, apresentando uma média semanal de 1,91 e $2,87 \mu \mathrm{g} \mathrm{m}^{-2} \mathrm{~h}^{-1}$ para os sistemas cana queimada e cana crua, respectivamente. A maior perda de $\mathrm{N}_{2} \mathrm{O}$ para a atmosfera ocorreu de forma decrescente a partir de dois dias após a adubação nitrogenada aliada à cobertura dos resíduos no sistema cana crua. Já na área experimental de Campos dos Goytacazes, a aplicação de vinhaça potencializou o fluxo de $\mathrm{N}_{2} \mathrm{O}$ apresentando valores elevados (195 $\mu \mathrm{g} \mathrm{m}^{-2} \mathrm{~h}^{-1}$ ) cinco horas após a adubação. $\mathrm{O}$ tratamento sem aplicação de vinhaça apresentou valores de fluxo próximos a zero $\left(0,4 \mu \mathrm{g} \mathrm{m} \mathrm{m}^{-2} \mathrm{~h}^{-1}\right)$, na mesma coleta. Após quatro dias da adubação com vinhaça não houve mais diferença no fluxo de $\mathrm{N}_{2} \mathrm{O}$ entre os tratamentos avaliados. A quantidade de matéria orgânica facilmente assimilável para os microrganismos do solo, o aumento da umidade do solo devido à adição da vinhaça e a precipitação ocorrida logo após a adubação foram, provavelmente, as variáveis mais importantes no controle e na magnitude das emissões de $\mathrm{N}_{2} \mathrm{O}$.

Palavras-chave: Óxido nitroso; emissão de gases de efeito estufa; manejo da palha da cana; vinhaça.

\footnotetext{
* Universidade Federal Rural do Rio de Janeiro, Instituto de Agronomia, Departamento de Solos, CEP 23890-000, Seropédica-RJ - Brasil.

Merika.solos@gmail.com

DOI: $\underline{10.21577 / 1984-6835.20170113}$
}

Rev. Virtual Quim. |Vol 9| | No. 5| |1930-1943| 


\title{
Emissões de $\mathrm{N}_{\mathbf{2}} \mathrm{O}$ em Solos sob Cultivo de Cana-de-Açúcar no bioma Mata Atlântica: Efeito dos Sistemas de Colheita e da Adubação com Vinhaça
}

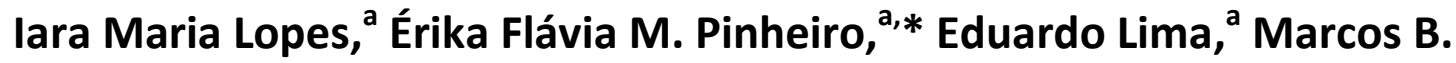 \\ Ceddia, ${ }^{a}$ David V. B. de Campos, ${ }^{b}$ Bruno José Alves ${ }^{c}$ \\ a Universidade Federal Rural do Rio de Janeiro, Instituto de Agronomia, Departamento de \\ Solos, CEP 23890-000, Seropédica-RJ - Brazil. \\ ${ }^{\mathrm{b}}$ Empresa Brasileira de Pesquisa Agropecuária - Embrapa Solos, Centro Nacional de Pesquisa \\ em Solos, Rua Jardim Botânico, 1024, CEP 22460-000 - Rio de Janeiro-RJ - Brazil. \\ ${ }^{\mathrm{C}}$ Empresa Brasileira de Pesquisa Agropecuária - Embrapa Agrobiologia, BR 465, km 7, \\ Seropédica, Rio de Janeiro-RJ, Brazil. \\ * erika.solos@gmail.com
}

Recebido em 28 de agosto de 2017. Aceito para publicação em 28 de agosto de 2017

\section{Introdução}

\section{Material e Métodos}

2.1. Descrição das áreas de estudo

2.2. Amostragem

2.3. Determinação da concentração de $\mathrm{N}_{2} \mathrm{O}$

2.4. Análise dos dados

\section{Resultados e Discussão}

3.1. Emissão de $\mathrm{N}_{2} \mathrm{O}$ e manejo da palhada da cana-de-açúcar em Linhares (ES)

3.2. Emissão de $\mathrm{N}_{2} \mathrm{O}$ e aplicação de vinhaça na cana-de-açúcar em Campos dos Goytacazes (RJ)

\section{Conclusões}

\section{Introdução}

O etanol de cana-de-açúcar é considerado a melhor alternativa para substituição de combustíveis fósseis, pois emite até $80 \%$ menos de gases de efeito estufa (GEE). ${ }^{1} \mathrm{O}$ Brasil é o maior produtor mundial de canade-açúcar (Saccharum spp L.) com, aproximadamente, 8,7 milhões de hectares de área cultivada. ${ }^{2}$ Atualmente, a queima dos canaviais tem sido cada vez menos comum, principalmente por questões ambientais. No estado de São Paulo, na safra de 2015/2016, cerca de 3,46 milhões de hectares (mais de $90 \%$ da área total) foram colhidos sem queima reduzindo a emissão de poluentes prejudiciais à saúde humana e a emissão de 
GEE responsáveis pelas mudanças climáticas. ${ }^{3}$ Contudo, nos estados do Espírito Santo e do Rio de Janeiro com, aproximadamente, 78 e 97 mil hectares de área com cana-de-açúcar, respectivamente, ${ }^{4}$ a abolição total da queima só está prevista para 2020 no estado do ES (Lei no 5.990/2011) $)^{5}$ e em 2024 no RJ (Lei no $9.073 / 2008)^{6}$. Assim, nesses dois estados ainda ocorre a queima da palhada em boa parte dos canaviais.

Essas áreas tradicionais no cultivo da cana-de-açúcar estão inseridas no bioma Mata Atlântica do Brasil, onde a agricultura e pecuária foram responsáveis por grande parte do desmatamento. A intensa atividade agropecuária se deve a inserção dessas áreas na feição geológica Tabuleiros costeiros, que se caracteriza por apresentar solos profundos e de baixa fertilidade, mas situados em regiões de topografia plana, tabulares, que facilita a mecanização. $O$ setor de mudança de uso da terra com a substituição da vegetação de mata nativa para áreas sob agricultura e/ou pecuária é o maior responsável pela emissão de GEE no país. No relatório feito pelo Ministério da Ciência, Tecnologia, Inovações e Comunicações (MCTIC), "Estimativas Anuais de Emissão de Gases de Efeito Estufa no Brasil", referentes ao período de 2010 a 2014, o setor mudanças de uso da terra e florestas mostrou uma queda de $33,2 \%$ na emissão de GEE. ${ }^{7}$ Além da diminuição dos desmatamentos, uma alternativa à redução de GEE é a mudança nas práticas agrícolas, como por exemplo, a redução da queimada. A adoção de práticas de manejo adequadas no sistema soloplanta-atmosfera, que visam à manutenção ou mesmo o acúmulo de carbono no solo contribuem na diminuição da emissão de GEE, o que pode resultar em um balanço geral (entrada $x$ saída) mais baixo e até mesmo negativo de emissão de GEE na agricultura, graças a menor emissão desses gases aliado ao maior estoque de $\mathrm{C}$ no solo. ${ }^{8,9}$

A queima da palhada é realizada com a finalidade de facilitar a colheita da cana-deaçúcar. Porém, a ausência da queimada resulta num aporte anual de 10 a $20 \mathrm{Mg} \mathrm{ha}^{-1}$ de massa seca de palhada sobre a superfície do solo. ${ }^{10}$ A preservação da palhada promove vários benefícios agronômicos, além de favorecer a retenção de água e promover o balanço de nutrientes no solo, também aumenta a diversidade de organismos no solo e mitiga a emissão de GEE, principalmente $\mathrm{CO}_{2}, \mathrm{~N}_{2} \mathrm{O}$ e $\mathrm{CH}_{4}$. Bordonal e colaboradores afirmam que a conversão do sistema com queima para o sem queima da palhada da cana-de-açúcar aliado ao menor revolvimento do solo são práticas responsáveis em diminuir a emissão de GEE. ${ }^{8}$ Esses autores, verificaram que a queima da palhada emite, em média, $883,6 \mathrm{~kg} \mathrm{ha}^{-1} \mathrm{ano}^{-1}$ de $\mathrm{CO}_{2}$ eq e deixa de acumular no solo 888,1 $\mathrm{kg} \mathrm{ha}^{-1} \mathrm{ano}^{-1}$ de $\mathrm{CO}_{2}$ eq. Além disso, os autores também observaram que o preparo reduzido do solo mitiga $201,7 \mathrm{~kg} \mathrm{ha}^{-1}$ ano $^{-1}$ de $\mathrm{CO}_{2} \mathrm{eq}$ quando comparado ao preparo convencional.

Dentre os GEEs, destaca-se o óxido nitroso que possui um potencial de aquecimento global 298 vezes maior que o $\mathrm{CO}_{2},{ }^{11}$ sendo responsável por, aproximadamente, $30 \%$ do total de GEE em $\mathrm{CO}_{2}$ eq emitido pelo setor agropecuário no ano de 2015 no Brasil. ${ }^{12} \mathrm{~A}$ conversão do uso da terra para sistemas agrícolas com queima dos resíduos agrícolas aumenta potencialmente a emissão de $\mathrm{N}_{2} \mathrm{O}$. ${ }^{13}$ $A$ emissão desse gás é oriunda dos processos microbiológicos que ocorrem nos solos: nitrificação e desnitrificação. A nitrificação é o processo de oxidação do nitrogênio na forma de amônio para nitrato sendo mediado por bactérias quimiotróficas aeróbias. A desnitrificação é o processo de redução biológica do $\mathrm{N}$-nitrato, ou N-nitrito, para $\mathrm{N}_{2} \mathrm{O}$, $\mathrm{NO}$ ou até mesmo $\mathrm{N}_{2}$. Esse processo ocorre simultaneamente ao processo de mineralização do N-orgânico no solo. ${ }^{14} \mathrm{~A}$ desnitrificação juntamente com a volatilização da amônia, constituem as mais importantes vias de perdas gasosas de nitrogênio no solo. Os principais fatores que interferem na emissão de $\mathrm{N}_{2} \mathrm{O}$ são: umidade, temperatura, presença de oxigênio, teores disponíveis de carbono orgânico e de nitrogênio e a relação $\mathrm{C} / \mathrm{N}$ do solo. ${ }^{15} \mathrm{~A}$ ocorrência natural desses processos nos solos de florestas tropicais e os estímulos realizados no manejo dos solos agrícolas pela larga adição de fertilizantes minerais 
nitrogenados resultam nestes ambientes como maiores fontes de emissão de $\mathrm{N}_{2} \mathrm{O}$. ${ }^{16}$ Além dos fertilizantes minerais, a adubação orgânica é outro fator responsável pela perda de $\mathrm{N}_{2} \mathrm{O}$. A aplicação de vinhaça, fertilizante orgânico produzido pelas usinas sucroalcooleiras, nas áreas de cana-de-açúcar através da irrigação é uma prática bastante adotada. Essa adubação tem sido realizada devido ao elevado conteúdo de potássio na vinhaça, porém, esse fertilizante orgânico estimula a produção de $\mathrm{N}_{2} \mathrm{O}$ através da saturação dos poros do solo e do aumento da oferta de Carbono lábil. Estudos realizados em áreas com aplicação de vinhaça, bem como nos canais escoadouros, têm comprovado que a vinhaça contribui substancialmente na emissão de GEE, em especial de $\mathrm{N}_{2} \mathrm{O}$ e $\mathrm{CH}_{4} \cdot{ }^{17-20}$

Segundo Oliveira e colaboradores, ${ }^{19}$ um volume aplicado de $200 \mathrm{~m}^{3} \mathrm{ha}^{-1}$ de vinhaça adiciona ao solo em torno de $46 \mathrm{Kg} \mathrm{ha}^{-1}$ de $\mathrm{N}$, o que aumenta a liberação de $\mathrm{N}_{2} \mathrm{O}$ resultando em fatores de emissão de 0,68 e 0,44 \% nas áreas com e sem queima da palha, respectivamente. Além disso, quando os fluxos de $\mathrm{N}_{2} \mathrm{O}$ e $\mathrm{CH}_{4}$ são convertidos em $\mathrm{CO}_{2}$ eq ha ${ }^{-1}$, a aplicação de $1 \mathrm{~m}^{3} \mathrm{ha}^{-1}$ de vinhaça emite 0,491 e 0,314 $\mathrm{kg}$ de $\mathrm{CO}_{2}$ eq nas áreas com e sem queima, respectivamente. Os fatores de emissão de $\mathrm{N}_{2} \mathrm{O}$ são calculados com base na diferença do somatório da emissão total de $\mathrm{N}_{2} \mathrm{O}$ da câmara nos tratamentos que recebem $\mathrm{N}$ mineral ou orgânico em relação ao somatório do tratamento controle (sem $\mathrm{N}$ mineral e orgânico) dividido pela quantidade de $\mathrm{N}$ aplicado na câmara. ${ }^{18}$

O objetivo do estudo foi quantificar a emissão de óxido nitroso sob diferentes sistemas de colheita e adubação com vinhaça, em duas áreas tradicionais no cultivo da cana-de-açúcar na região de Mata Atlântica do Brasil sendo uma área com diferentes sistemas de manejo da palhada perante a colheita (cana queimada e cana crua) em Linhares-ES e a outra com diferentes manejos de adubação orgânica (com e sem aplicação de vinhaça) em Campos dos Goytacazes-RJ.

\section{Material e Métodos}

\subsection{Descrição das áreas de estudo}

O trabalho foi conduzido em duas áreas experimentais. Uma área experimental pertencente à LAGRISA (Linhares Agrícola S/A), no município de Linhares, no Estado do Espírito Santo. O solo da área experimental é classificado como Argissolo Amarelo Distrófico, com textura arenosa. O experimento foi instalado em 28/05/1989 visando um estudo de longa duração dos sistemas de corte da cana-de-açúcar. Para tal, após a retirada da mata secundária fez-se o plantio da variedade de cana RB 73-9735 que apresenta porte ereto e fácil despalha, características que facilitam o corte e a colheita da cana em sistema sem queima.

A adubação no plantio foi de $500 \mathrm{~kg} \mathrm{ha}^{-1}$ do adubo formulado 0,4-20-20, sendo $2 \mathrm{~kg}$ $\mathrm{ha}^{-1}$ de $\mathrm{N}$ na forma de ureia, $100 \mathrm{~kg} \mathrm{ha}^{-1} \mathrm{de}$ $\mathrm{K}_{2} \mathrm{O}$ na forma de cloreto de potássio e $100 \mathrm{~kg}$ ha ${ }^{-1}$ de $\mathrm{P}_{2} \mathrm{O}_{5}$ na forma de superfosfato simples, respectivamente. Nos anos seguintes (1990-1996), sempre após as colheitas, foi realizada a adubação das soqueiras com $500 \mathrm{~kg} \mathrm{ha}^{-1}$ do adubo formulado 20-0-30, sendo $100 \mathrm{~kg} \mathrm{ha}^{-1}$ de $\mathrm{N}$ na forma de ureia e $150 \mathrm{~kg} \mathrm{ha}^{-1}$ de $\mathrm{K}_{2} \mathrm{O}$ na forma de cloreto de potássio, distribuídos uniformemente sobre a palhada.

Os tratamentos avaliados foram: a) corte da cana após a queima da palhada (cana queimada - CQ) e b) corte da cana sem a queima da palhada e com distribuição desta na superfície do solo (cana crua - CC) (Figura $1)$.

A outra área experimental foi conduzida na Estação Experimental da UFRRJ no Campus Leonel Miranda em Campos dos Goytacazes, no Estado do Rio de Janeiro. A área experimental vem sendo cultivada com cana-de-açúcar desde 1981. No momento da 
instalação do experimento a área estava cultivada com cana da variedade RB85- 5536. $\mathrm{O}$ solo é classificado como Cambissolo. A adubação no plantio foi de $500 \mathrm{~kg} \mathrm{ha}^{-1}$ do adubo formulado $0,4-20-20$, sendo $2 \mathrm{~kg} \mathrm{ha}^{-1}$ de $\mathrm{N}$ na forma de ureia, $100 \mathrm{~kg} \mathrm{ha}^{-1}$ de $\mathrm{K}_{2} \mathrm{O}$ na forma de cloreto de potássio e $100 \mathrm{~kg} \mathrm{ha}^{-1} \mathrm{de}$ $\mathrm{P}_{2} \mathrm{O}_{5}$ na forma de superfosfato simples, respectivamente. Nos anos seguintes, após as colheitas, foram realizadas a adubação das soqueiras com $500 \mathrm{~kg} \mathrm{ha}^{-1}$ do adubo formulado 20-0-30, sendo $100 \mathrm{~kg} \mathrm{ha}^{-1}$ de $\mathrm{N}$ na forma de ureia e $150 \mathrm{~kg} \mathrm{ha}^{-1}$ de $\mathrm{K}_{2} \mathrm{O}$ na forma de cloreto de potássio, distribuídos uniformemente.

Os tratamentos avaliados foram: a) cana adubada com vinhaça (CV) - nesse tratamento foi realizada a adubação orgânica da cana-de-açúcar com uma única aplicação de $150 \mathrm{~m}^{3} \mathrm{ha}^{-1}(0,2 \%$ de $\mathrm{N})$ de vinhaça e, b) cana sem adubação com vinhaça (SV) - nesse tratamento não foi aplicada a vinhaça como adubo orgânico.
A

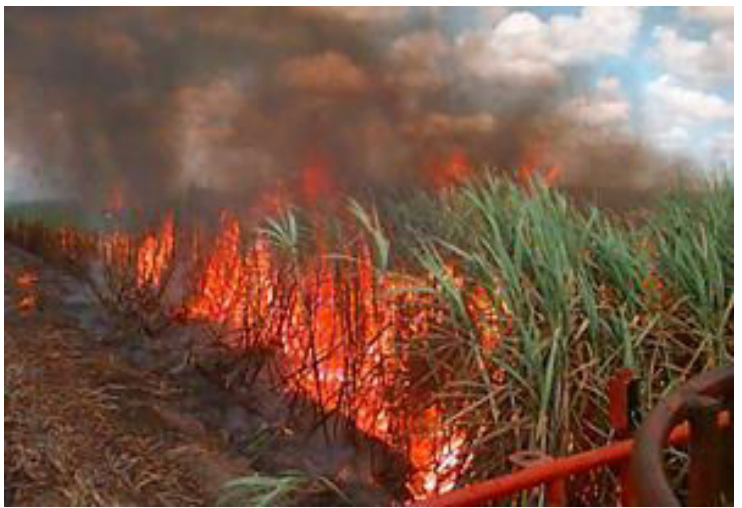

C

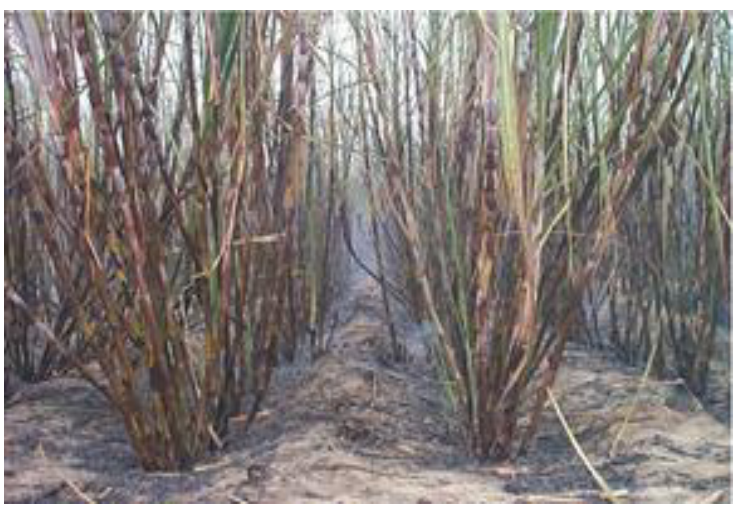

B

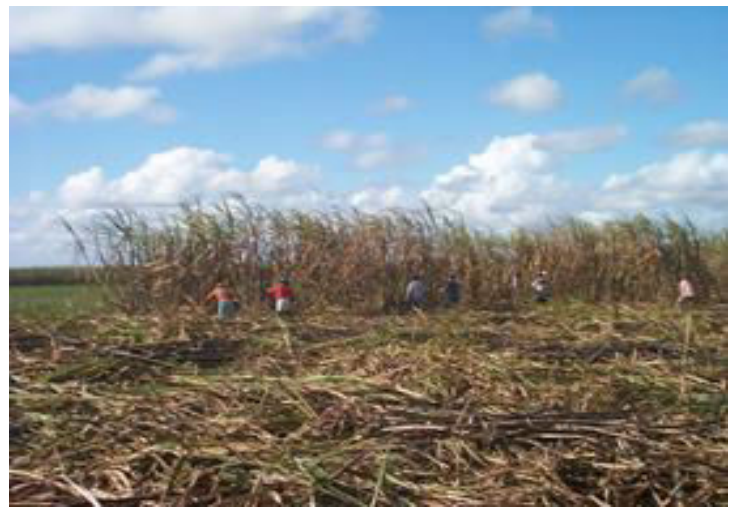

D

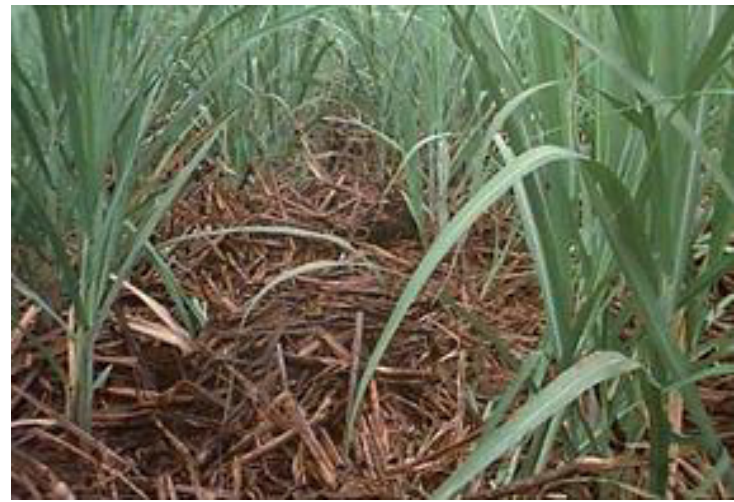

Figura 1. Detalhe dos sistemas de colheita cana queimada e crua (A e B) e do respectivo aporte de palhada na superfície do solo (C e D)

O delineamento experimental foi o de blocos ao acaso, com seis repetições para cada tratamento em ambas as áreas de estudo (Linhares-ES e Campos dos Goytacazes-RJ).

\subsection{Amostragem}

Nas duas áreas de estudo foram instaladas câmaras estáticas especialmente construídas em cada parcela experimental para a amostragem do fluxo de $\mathrm{N}_{2} \mathrm{O}$. As câmaras foram posicionadas na linha de plantio da cana-de-açúcar. Cada câmara era composta por uma base retangular de $30 \times 25 \mathrm{~cm}$ de metal, inserida no solo até $5 \mathrm{~cm}$ de profundidade (Figura 2). Em cada amostragem, a base era coberta com uma tampa de metal com as mesmas dimensões de largura e espessura da base de metal, e selada com grampos de carpinteiro. 
As amostras foram coletadas com auxílio de seringas de nylon de $20 \mathrm{~mL}$ sendo o gás diretamente transferido para frascos (selados com rolhas de borracha butírica, recravados com lacres de alumínio) previamente submetidos a vácuo. $O$ vácuo foi realizado com auxílio de um vacuômetro manual até atingir uma pressão de $-80 \mathrm{Kpa}$. $\mathrm{O}$ intervalo entre a coleta e a análise dos gases não ultrapassou dez dias pois, testes realizados na Embrapa Agrobiologia, com padrão de 500 ppbv de $\mathrm{N}_{2} \mathrm{O}$ demonstraram que os frascos utilizados mantinham a concentração inicial do gás por até 15 dias. ${ }^{21}$ No momento da coleta do gás também foram determinadas a temperatura do solo e do ar com auxílio de um termômetro digital. As medidas foram realizadas uma vez ao dia sempre no período da manhã.

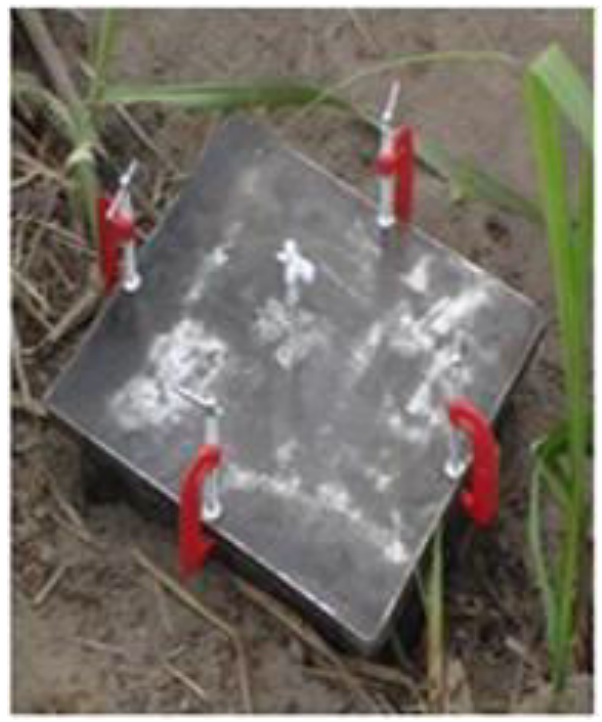

Figura 2. Detalhe da câmara instalada na linha de plantio da cana-de-açúcar e do coletor do gás na parte central da tampa

Em Linhares-ES retirou-se uma amostra do gás $\mathrm{N}_{2} \mathrm{O}$ um dia antes e cinco dias após a adubação mineral, no período entre 22 e 27 de outubro de 2007. Para a coleta do gás foi retirada uma amostra inicial, logo após o fechamento da câmara $\left(t_{0}\right)$ e após 15 minutos de fechamento da câmara $\left(t_{1}\right)$.

Em Campos dos Goytacazes-RJ também foi feita uma coleta do gás um dia antes da adubação orgânica com vinhaça (4 de julho de 2008). No entanto, a segunda coleta ocorreu, excepcionalmente, no mesmo dia (04/07/08) no período da tarde (cinco horas após a adubação) devido à elevada precipitação ocorrida. As demais amostragens do gás $\mathrm{N}_{2} \mathrm{O}$ ocorreram nos dias 5, 7 e 8 de julho de 2008.

Imediatamente após a coleta do gás $\mathrm{N}_{2} \mathrm{O}$ foi avaliada a umidade do solo (U), a densidade do solo (ds) e a densidade da partícula do solo (dp) na camada superficial do solo $(0-20 \mathrm{~cm}$ em Linhares-ES e $0-10 \mathrm{~cm}$ em Campos dos Goytacazes-RJ), segundo a metodologia da Embrapa. ${ }^{22}$ Posteriormente foi calculado o espaço poroso saturado por água (\% EPSA = ( $U \mathrm{x} d \mathrm{ds}) / 1-(\mathrm{ds} / \mathrm{dp}))$, em que: $U$ é a umidade gravimétrica do solo $\left(\mathrm{g} \mathrm{g}^{-1}\right)$; ds é a densidade do solo $\left(\mathrm{g} \mathrm{cm}^{-3}\right)$ e dp é a densidade de partículas do solo $\left(\mathrm{g} \mathrm{cm}^{-3}\right)$, conforme proposto por Paul \& Clark. ${ }^{23}$

\subsection{Determinação da concentração de} $\mathrm{N}_{2} \mathrm{O}$

Os frascos com as amostras do gás $\mathrm{N}_{2} \mathrm{O}$ 
foram enviados para análise no Laboratório de Solos da Embrapa Agrobiologia. Antes de cada conjunto de análises, foram injetadas amostras do padrão de concentração conhecida de $\mathrm{N}_{2} \mathrm{O}$ (312 ppbv, 400 ppbv, 800 ppbv, 1120 ppbv e 10000 ppbv) para o cálculo das concentrações de $\mathrm{N}_{2} \mathrm{O}$ em cada amostra. A concentração de $\mathrm{N}_{2} \mathrm{O}$ foi determinada por cromatografia gasosa (Perkin Elmer, Auto system $\mathrm{XL}$, Wellesley, MA, USA), utilizando-se uma coluna empacotada com Porapak $Q$ e um detector de captura de elétrons. Os fluxos de $\mathrm{N}_{2} \mathrm{O}$ foram calculados pela equação: $\mathrm{N}_{2} \mathrm{O}=$ $\delta \mathrm{C} / \delta \mathrm{t} \times \mathrm{V} / \mathrm{A} \times \mathrm{M} / \mathrm{Vm}^{24}{ }^{24}$ em que: o fluxo de $\mathrm{N}_{2} \mathrm{O}$ é expresso em $\mu \mathrm{g} \mathrm{m}^{-2} \mathrm{~h}^{-1} ; \delta \mathrm{C} / \delta \mathrm{t}$ é a variação da concentração de $\mathrm{N}_{2} \mathrm{O}$ (ppbv), entre $t_{0}$ e $t_{1}$ (hora); $V$ é o volume da câmara $\left(\mathrm{m}^{3}\right)$; $A$ é a área da câmara $\left(\mathrm{m}^{2}\right)$; $M$ é a massa atômica de $\mathrm{N}$ na molécula de $\mathrm{N}_{2} \mathrm{O}(\mathrm{mg})$; e $\mathrm{Vm}$ é o volume molecular na temperatura de amostragem $\left(\mathrm{dm}^{3}\right)$.

\subsection{Análise dos dados}

As comparações entre os tratamentos cana queimada e cana crua no experimento em Linhares (ES) e entre a cana adubada ou não com vinhaça foram realizadas através da análise de variância com aplicação do teste $\mathrm{F}$ $(p<0,05)$, utilizando o programa estatístico Sistema para Análises Estatísticas e Genéricas (SAEG), versão 5.0. ${ }^{25}$ Também foram apresentados os resultados do erro-padrão da média, considerando as seis repetições ( $\mathrm{n}$ $=6)$.

\section{Resultados e Discussão}

\subsection{Emissão de $\mathrm{N}_{2} \mathrm{O}$ e manejo da palhada da cana-de-açúcar em Linhares-ES}

Os valores de fluxo de $\mathrm{N}_{2} \mathrm{O}$ comparando os diferentes sistemas de colheita da cana-deaçúcar estão apresentados na Figura 2. Podese observar que as emissões de $\mathrm{N}_{2} \mathrm{O}$ não foram elevadas e não diferiram estatisticamente entre os tratamentos avaliados, apresentando uma média semanal de 1,91 e $2,87 \mu \mathrm{g} \mathrm{m}^{-2} \mathrm{~h}^{-1}$ de $\mathrm{N}_{2} \mathrm{O}$ para os sistemas de colheita cana queimada e cana crua, respectivamente. Um dia antes da adubação, o sistema de manejo sem a queima do canavial apresentou os menores fluxos de $\mathrm{N}_{2} \mathrm{O}$ quando comparado com o sistema cana queimada (Figura 3 ).

A maior perda de $\mathrm{N}_{2} \mathrm{O}$ ocorreu três dias após a adubação no sistema cana crua (dia $25 / 10$ ), sendo estatisticamente significativa a diferença entre os sistemas de colheita $(p<0,05)$. O uso do fertilizante nitrogenado aliado à cobertura dos resíduos culturais resultou em maiores emissões de $\mathrm{N}_{2} \mathrm{O}$ do solo para a atmosfera no sistema cana crua.

Estudos comprovam que as maiores emissões de $\mathrm{N}_{2} \mathrm{O}$ no solo se concentram após a adubação nitrogenada nos períodos de maiores precipitações. ${ }^{26,27}$ Quando os solos não apresentam condições limitantes de disponibilidade de $\mathrm{N}$ e o espaço poroso saturado por água é maior que $60 \%,{ }^{28}$ ocorre o predomínio do processo de desnitrificação, onde $\mathrm{NO}_{3}{ }^{-}$é reduzido a $\mathrm{N}_{2} \mathrm{O}$. 


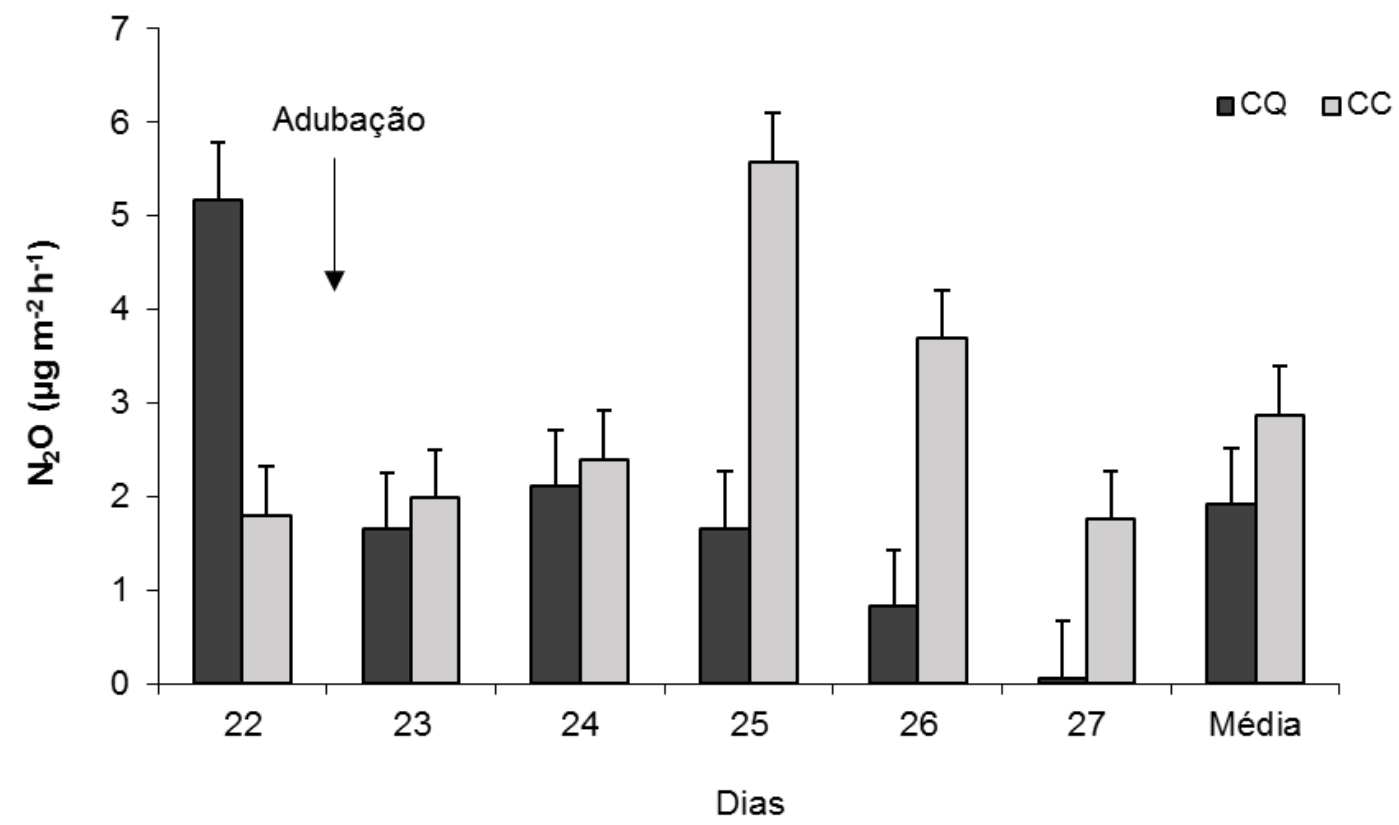

Figura 3. Fluxo de $\mathrm{N}_{2} \mathrm{O}\left(\mu \mathrm{g} \mathrm{m}^{-2} \mathrm{~h}^{-1}\right)$ antes e após a adubação sob diferentes sistemas de colheita da cana-de-açúcar, em Linhares (ES). CQ: cana queimada e CC: cana crua (sem queima). As barras verticais, em cada avaliação, representam o erro padrão da média $(n=6)$

O espaço poroso saturado por água (EPSA) médio, referente aos dias de coleta do gás, não diferiram estatisticamente $(p<0,05)$ entre os sistemas de colheita da cana-de-açúcar (Figura 4). Esse resultado está provavelmente associado ao curto espaço de tempo de implantação dos tratamentos (com queima e sem queima) e também devido à ausência de chuva no período de avaliação. Os fluxos de $\mathrm{N}_{2} \mathrm{O}$ verificados nesse estudo, considerados relativamente baixos, podem estar relacionados ao baixo EPSA e a textura arenosa do solo Argissolo Amarelo que promovem uma baixa retenção de água nesse solo.

Os maiores fluxos de $\mathrm{N}_{2} \mathrm{O}$ nas áreas de cana-de-açúcar sem queima e com a manutenção de toda a palhada sobre o solo ocorrem em razão da palhada conservar a umidade do solo por mais tempo e, além disso, por beneficiar a atividade microbiana do solo, resultando em maior decomposição da palhada e consequentemente maior liberação de $C$ solúvel que favorece 0 processo de desnitrificação. ${ }^{20,29}$ Assim, a manutenção da palhada sobre o solo, principalmente, quando em maiores quantidades aumenta a emissão de $\mathrm{N}_{2} \mathrm{O}$ quando comparado ao tratamento sem palha. ${ }^{20,29}$

Num estudo realizado por Carmo e colaboradores $^{20}$ no município de Piracicaba$\mathrm{SP}$, com diferentes deposições de palha da cana-de-açúcar sobre o solo $(0,7,14$ e $21 \mathrm{Mg}$ $\mathrm{ha}^{-1}$ ) e com e sem a aplicação de vinhaça (56 $\left.\mathrm{m}^{3} \mathrm{ha}^{-1}\right)$, os autores observaram que na área sem vinhaça a aplicação do adubo mineral potencializou a emissão de $\mathrm{N}_{2} \mathrm{O}$, em especial no tratamento com maior quantidade de palhada sobre o solo (4000 $\mathrm{g} \mathrm{ha}^{-1} \mathrm{ano}^{-1} \mathrm{de}$ $\mathrm{N}_{2} \mathrm{O}$ ), sendo praticamente o dobro do valor emitido no tratamento sem palha (aproximadamente $2000 \mathrm{~g} \mathrm{ha}^{-1} \mathrm{ano}^{-1}$ de $\mathrm{N}_{2} \mathrm{O}$ ). Os autores verificaram ainda que no tratamento sem palha e sem adubação os valores emitidos ficaram próximos de zero. 


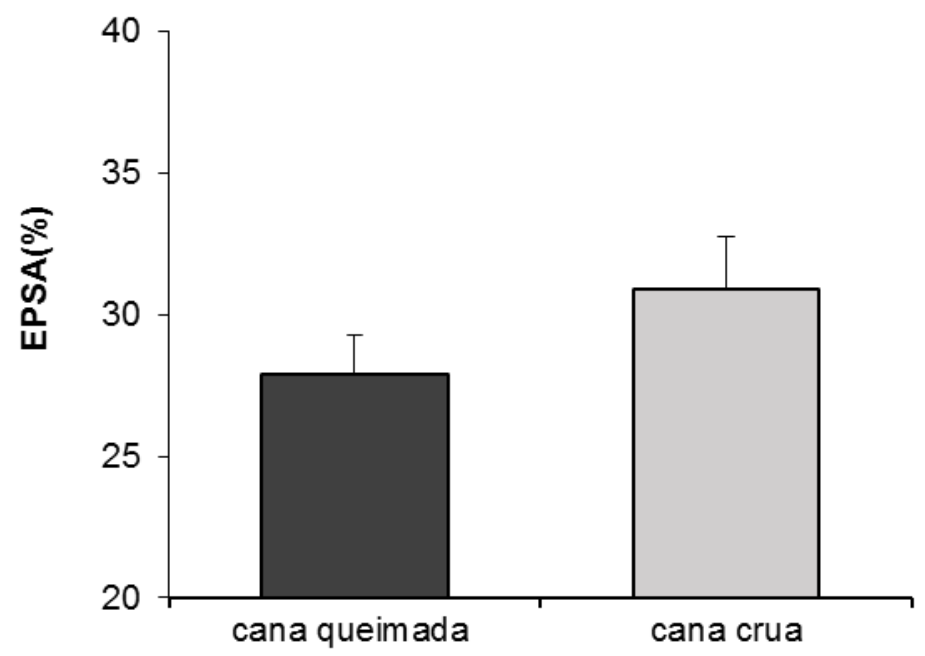

Figura 4. Espaço poroso saturado por água -EPSA (\%) na camada de 0-20 cm, nos diferentes sistemas de colheita da cana-de-açúcar em um Argissolo Amarelo, em Linhares (ES). As barras verticais representam o erro padrão da média $(n=6)$

Constatou-se no presente estudo que os fluxos de $\mathrm{N}_{2} \mathrm{O}$ apresentaram elevada variabilidade, refletidos através dos coeficientes de variação. Observou-se ainda fluxos negativos de $\mathrm{N}_{2} \mathrm{O}$ (i.e., fluxos de $\mathrm{N}_{2} \mathrm{O}$ da atmosfera para o solo). Os valores variaram entre 7,52 e 11,74 $\mu \mathrm{g} \mathrm{m}^{-2} \mathrm{~h}^{-1}$ de $\mathrm{N}_{2} \mathrm{O}$. Vários trabalhos também observaram fluxos negativos de $\mathrm{N}_{2} \mathrm{O}$, esses fluxos são frequentes e substanciais e não podem ser simplesmente atribuídos ao erro experimental. ${ }^{30-32}$

$\mathrm{Na}$ literatura, Blackmer \& Bremner mostraram que alguns solos têm maior capacidade de capturar $\mathrm{N}_{2} \mathrm{O}$ da atmosfera do que liberá-lo, ${ }^{30}$ o que sustenta fortemente essa hipótese do solo como fonte de $\mathrm{N}_{2} \mathrm{O}$ proveniente da atmosfera. Aproximadamente $18 \%$ dos valores de fluxo de $\mathrm{N}_{2} \mathrm{O}$ observados nesse estudo foram negativos, apesar de não apresentar nenhuma média com valor negativo (Figura 3).

Os valores de fluxos de $\mathrm{N}_{2} \mathrm{O}$ negativos foram reportados por diversos trabalhos, os quais relatam que os estudos disponíveis não enfatizam esses dados como sendo uma propriedade importante em vários sistemas. ${ }^{31,32} \mathrm{Em}$ torno de $30-40 \%$ dos dados observados durante uma coleta de gás $\mathrm{N}_{2} \mathrm{O}$ numa estação úmida na Amazônia brasileira foram negativos. Esses fluxos negativos de $\mathrm{N}_{2} \mathrm{O}$ estão relacionados a baixas concentrações de $\mathrm{N}$ inorgânico, oxigênio, conteúdo de água e temperatura do solo. ${ }^{31,32}$

\subsection{Emissão de $\mathrm{N}_{2} \mathrm{O}$ e aplicação de vinhaça na cana-de-açúcar em Campos dos Goytacazes-RJ}

Os valores de fluxo de $\mathrm{N}_{2} \mathrm{O}$ comparando a aplicação ou não de vinhaça no canavial estão apresentados na Figura 2. Como resultados, pode-se observar que antes de adubar com vinhaça, no tempo inicial (dia 4), os valores de fluxos de $\mathrm{N}_{2} \mathrm{O}$ eram baixos, próximos a zero. Entretanto, um dia após a adubação (dia 4) os valores de fluxo de $\mathrm{N}_{2} \mathrm{O}$ foram bastante elevados no tratamento que recebeu a aplicação de vinhaça $\left(150 \mathrm{~m}^{3} \mathrm{ha}^{-1}\right)$. Este tratamento apresentou um fluxo de 195 $\mu \mathrm{g} \mathrm{m} \mathrm{m}^{-2} \mathrm{~h}^{-1}$ de $\mathrm{N}_{2} \mathrm{O}$ cinco horas após a aplicação da vinhaça sendo estatisticamente significativo $(p=0,05)$ ao tratamento sem aplicação de vinhaça que apresentou valores

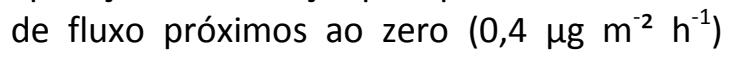
nesta mesma coleta (Figura 5). Esse resultado 
corrobora com o estudo de Siqueira Neto e colaboradores, os quais também observaram um valor próximo desse $\left(200 \mu \mathrm{g} \mathrm{m}^{-2} \mathrm{~h}^{-1}\right)$ porém, somente dois dias após a aplicação de $150 \mathrm{~m}^{3}$ ha $^{-1}$ sobre a palha da cana-de-açúcar em Piracicaba-SP. ${ }^{18}$

A maior perda de $\mathrm{N}_{2} \mathrm{O}$ foi observada cinco horas após a adubação com vinhaça (dia 4). No segundo dia após a adubação (dia 7) já se observou uma acentuada queda do fluxo de $\mathrm{N}_{2} \mathrm{O}$ e a partir desse dia não houve nenhuma diferença no fluxo de $\mathrm{N}_{2} \mathrm{O}$ entre os tratamentos avaliados, passando a valores negativos no dia 8 (Figura 5). Como base na média do fluxo de $\mathrm{N}_{2} \mathrm{O}$ no período avaliado, pode-se observar que a adubação com vinhaça estimulou o fluxo de $\mathrm{N}_{2} \mathrm{O}(38,15 \mu \mathrm{g}$ $\mathrm{m}^{-2} \mathrm{~h}^{-1}$ ) em relação à cana não adubada com vinhaça $\left(-2,46 \mu \mathrm{g} \mathrm{m}^{-2} \mathrm{~h}^{-1}\right)$ (Figura 5).

Siqueira Neto e colaboradores, ${ }^{18}$ ao compararem o fluxo médio de $\mathrm{N}_{2} \mathrm{O}$ (trinta dias de avaliação) após a aplicação de diferentes quantidades de vinhaça (150 e 300 $\mathrm{m}^{3} \mathrm{ha}^{-1}$ ) sobre a palha da cana-de-açúcar e $150 \mathrm{~m}^{3} \mathrm{ha}^{-1}$ de vinhaça associada a adubação mineral com $80 \mathrm{Kg} \mathrm{ha}^{-1}$ de $\mathrm{N}$ (fonte: ureia) em relação ao controle (sem vinhaça e sem adubação mineral), em Piracicaba-SP, observaram fluxos médios de $\mathrm{N}_{2} \mathrm{O}$ de 97,3 , $125,5,212,6$ e $41,9 \mu \mathrm{g} \mathrm{m} \mathrm{m}^{-2} \mathrm{~h}^{-1}$ para os tratamentos $150,300 \mathrm{~m}^{3} \mathrm{ha}^{-1}$ de vinhaça, 150 $\mathrm{m}^{3} \mathrm{ha}^{-1}$ de vinhaça mais $80 \mathrm{Kg} \mathrm{ha}^{-1}$ de $\mathrm{N}$ e controle, respectivamente. $\mathrm{O}$ que comprova que a aplicação de vinhaça nos canaviais, sobretudo de vinhaça e $\mathrm{N}$ mineral, aumenta em $507 \%$ a emissão de $\mathrm{N}_{2} \mathrm{O}$ em relação ao controle (sem vinhaça e sem adubação mineral).

Em outro estudo no município de Piracicaba-SP, com aplicação de vinhaça (56 $\left.\mathrm{m}^{3} \mathrm{ha}^{-1}\right)$ sobre diferentes deposições de palha da cana-de-açúcar sobre o solo $(0 ; 7 ; 14$ e 21 $\mathrm{Mg} \mathrm{ha}{ }^{-1}$ ) e com tratamento controle sem palha e sem adubação mineral ou orgânica, Carmo e colaboradores, ${ }^{20}$ verificaram que a aplicação de adubação mineral e principalmente a adubação orgânica (vinhaça) potencializa bastante a emissão de $\mathrm{N}_{2} \mathrm{O}$. Os valores observados na área com 21 $\mathrm{Mg} \mathrm{ha}^{-1}$ de palha e com vinhaça foi em torno de $7.400 \mathrm{~g} \mathrm{ha}^{-1}$ ano $^{-1}$ de $\mathrm{N}_{2} \mathrm{O}$, ou seja, $85 \%$ maior que a área com $21 \mathrm{Mg} \mathrm{ha}{ }^{-1}$ de palha porém sem vinhaça $\left(4.000 \mathrm{~g} \mathrm{ha}^{-1} \mathrm{ano}^{-1} \mathrm{de}\right.$ $\mathrm{N}_{2} \mathrm{O}$ ). Oliveira e colaboradores, ${ }^{17}$ também observaram que a aplicação de vinhaça (200 $\mathrm{m}^{3} \mathrm{ha}^{-1}$ ) principalmente nas áreas sem queima da palha em relação as áreas com queima, potencializam a emissão de $\mathrm{N}_{2} \mathrm{O}$, resultando em fatores de emissão de 0,68 e $0,44 \%$, nas áreas com e sem queima da palha, respectivamente.

As emissões de $\mathrm{N}_{2} \mathrm{O}$ em solos agrícolas estão fortemente associadas à fertilização nitrogenada. Trabalhos realizados em condições de clima temperado mostram uma relação linear entre a quantidade de fertilizante nitrogenado aplicado e as emissões de óxido nitroso. ${ }^{36,37}$ Observa-se também que o manejo da palha sobre o solo, a aplicação de vinhaça e $\mathrm{N}$ mineral, favorecem a maior emissão de $\mathrm{N}_{2} \mathrm{O}$ nos sistemas de produção de cana-de-açúcar. Contudo, é importante ressaltar que áreas que mantém a palha da cana-de-açúcar sobre o solo o balanço geral de emissão de GEE são mais baixos em aproximadamente $2.000 \mathrm{~kg}$ $\mathrm{ha}^{-1}$ ano $^{-1}$ de $\mathrm{CO}_{2}$ eq, graças ao sequestro de $\mathrm{C}$ no solo e a menor emissão pela ausência da queima. ${ }^{7,8,33-35}$ 


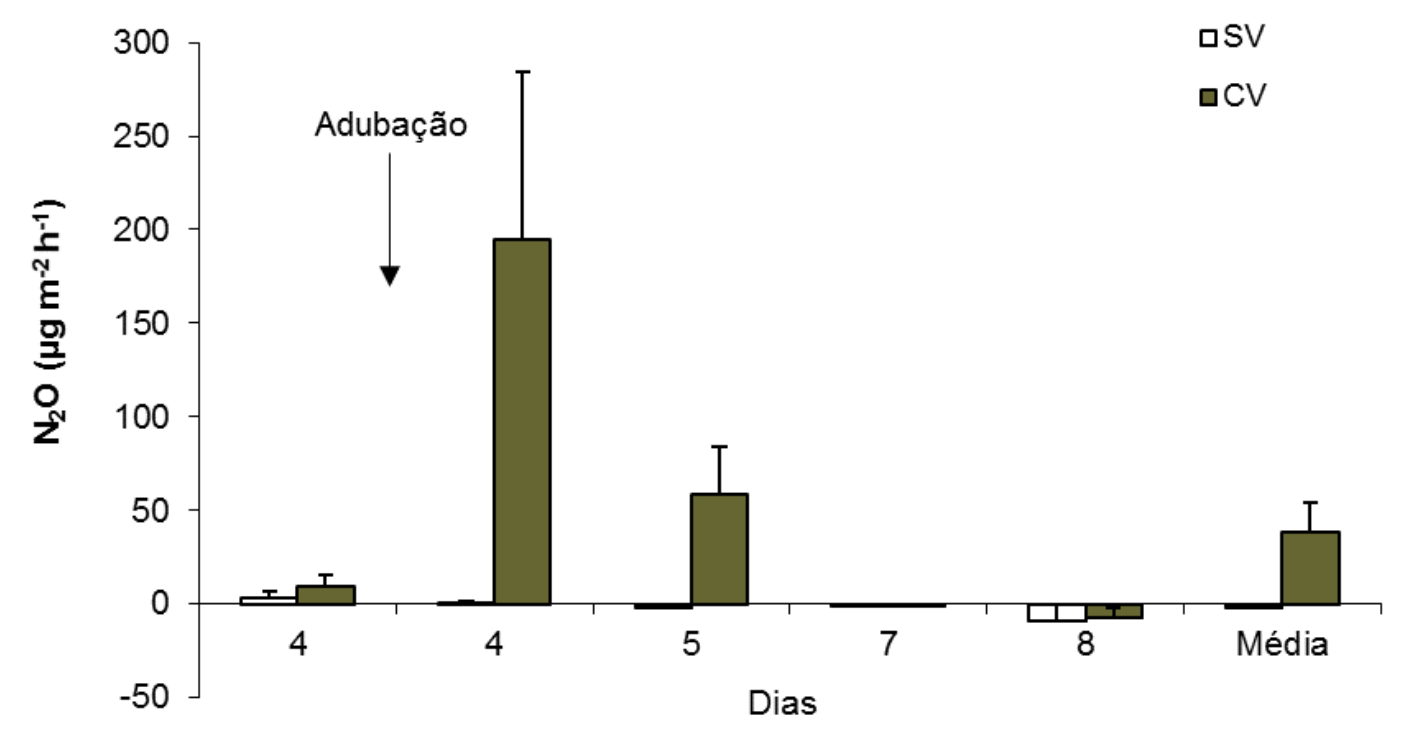

Figura 5. Fluxo de $\mathrm{N}_{2} \mathrm{O}\left(\mu \mathrm{gm}^{-2} \mathrm{~h}^{-1}\right)$ sob cobertura de cana-de-açúcar com e sem a adubação com vinhaça em Cambissolo, em Campos dos Goytacazes (RJ). SV: sem vinhaça; CV: com vinhaça. As barras verticais, em cada avaliação, representam o erro padrão da média $(n=6)$

A umidade do solo (grau de saturação de água no solo) causa grande influência nas emissões de $\mathrm{N}_{2} \mathrm{O}$. No primeiro dia após adubação com vinhaça, quando foi observada a maior perda de $\mathrm{N}_{2} \mathrm{O}$, o solo sob cana adubada com vinhaça apresentava, aproximadamente, $89 \%$ do espaço poroso saturado com água (EPSA) (Figura 6). Além da vinhaça adicionada ao solo o que também contribuiu para a elevação da umidade do solo foi a precipitação ocorrida. O solo sob cana sem adubação com vinhaça apresentou 58\% EPSA. Em condições de solo com baixos valores de espaço poroso do solo saturado com água, a nitrificação é processo responsável pela emissão de $\mathrm{N}_{2} \mathrm{O}$. Em condições de alta saturação do solo com água, a desnitrificação passa a ser o processo mais importante. Os solos com saturação dos poros com água inferior a $60-65 \%$, os fluxos de $\mathrm{N}_{2} \mathrm{O}$ são bastante baixos, sendo os maiores fluxos, observados em níveis de saturação próximos de $80-85 \% .{ }^{37,38}$

A umidade do solo pode explicar as maiores perdas de $\mathrm{N}_{2} \mathrm{O}$ ocorridas sob canade-açúcar após a adubação com vinhaça. Além disso, o teor de nitrogênio na vinhaça utilizada para a adubação da cana foi muito baixo, $0,2 \%$ N. Como os organismos quimiorganotróficos, em condições da anaerobiose, utilizam formas oxidadas de nitrogênio para a oxidação do carbono, a disponibilidade de carbono lábil é essencial para a ocorrência do processo. 


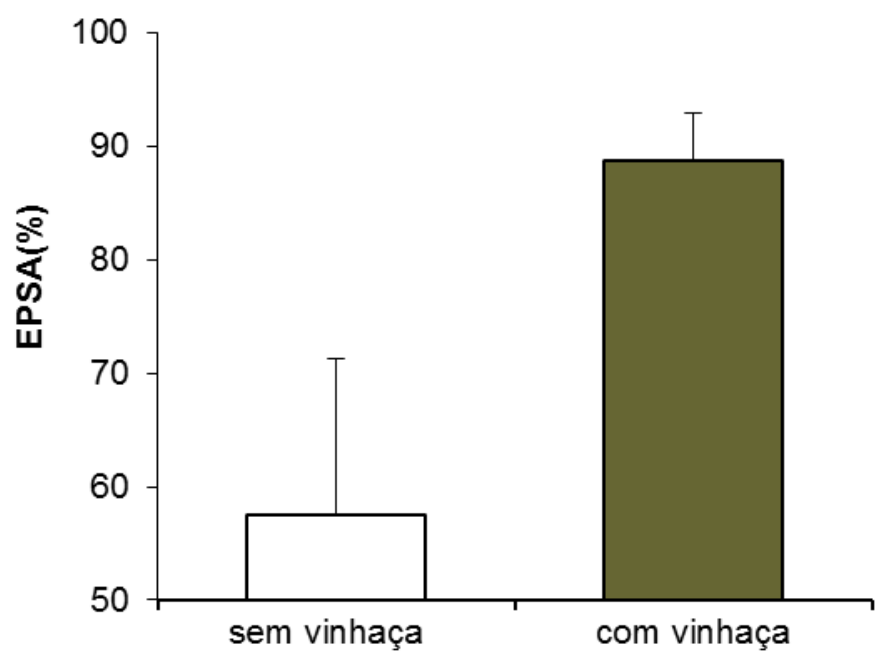

Figura 6. Espaço poroso saturado por água (EPSA) (\%), na camada de 0-10 cm, em área com cana-de-açúcar com e sem adubação com vinhaça, em outubro de 2007, em Campos dos Goitacazes (RJ). As barras verticais representam o erro padrão da média $(n=6)$

\section{Conclusões}

A manutenção da palhada da cana-deaçúcar sobre a superfície do solo, bem como a adubação com vinhaça, favoreceu maiores emissões de $\mathrm{N}_{2} \mathrm{O}$ nos sistemas de produção de cana-de-açúcar, na região de Mata Atlântica do Brasil.

O fluxo de $\mathrm{N}_{2} \mathrm{O}$ na área experimental localizada em Linhares (ES) apresentou valores decrescentes a partir do terceiro dia após aplicação do fertilizante mineral, o que refletiu num valor médio semanal de 1,91 e $2,87 \mu \mathrm{g} \mathrm{m}^{-2} \mathrm{~h}^{-1}$ para os sistemas cana queimada e cana crua, respectivamente.

A maior emissão de $\mathrm{N}_{2} \mathrm{O}$ em Campos dos Goytacazes ocorreu cinco horas após a aplicação de vinhaça (195 $\mu \mathrm{g} \mathrm{m}^{-2} \mathrm{~h}^{-1}$ de $\left.\mathrm{N}_{2} \mathrm{O}\right)$, apresentando uma redução três dias depois da adubação. Dessa forma, conclui-se que o uso da vinhaça nos canaviais deve ser feito com cautela, principalmente, nos períodos chuvosos, visando evitar maiores perdas de nitrogênio do solo para a atmosfera.

\section{Agradecimentos}

Os autores agradecem aos técnicos Geraldo, Gilson e Carminto da Estação Experimental da UFRRJ em Campos dos Goytacazes (Campus Leonel Miranda), à Usina LASA, ao CPGA-CS, a FAPERJ, a CAPES, o CNPq e a Embrapa Agrobiologia pelo apoio técnico e financeiro.

\section{Referências Bibliográficas}

${ }^{1}$ Macedo, I. C.; Seabra, J. E. A.; Silva, J. E. A. R. Greenhouse gases emissions in the production and use of ethanol from sugarcane in Brazil: The 2005/2006 averages and a prediction for 2020. Biomass and Bioenegy 2008, 32, 582. [CrossRef]

${ }^{2}$ Companhia Nacional de Abastecimento CONAB. Área Plantada de Cana-de-Açúcar, maio, 2017. [Link]

${ }^{3}$ Etanol Verde. Relatório da Safra 2015/2016 do Protocolo Agroambiental do Setor Sucroenergético, 2016.[Link] 
${ }^{4}$ CANASAT - Mapeamento da cana via imagens de satélite de observação da Terra, 2017. [Link]

${ }^{5}$ Lei 5990/11, de 20 de junho de 2011. Dispõe sobre a eliminação gradativa da queima da palha da cana-de-açúcar e dá outras providências no estado do Rio de Janeiro. [Link]

${ }^{6}$ Lei no 9.073, 2008, de 05 de dezembro de 2008. Dispõe sobre a eliminação gradativa da prática de queimadas nas colheitas de canade-açúcar e dá outras providências no estado do Espírito Santo. [Link]

${ }^{7}$ Ministério da Ciência, Tecnologia, Inovações e Comunicações (MCTIC): "Estimativas Anuais de Emissão de Gases de Efeito Estufa no Brasil", referentes ao período de 2010 a 2014.Disponível

em:

$<$ http://www.brasil.gov.br/ciencia-etecnologia/2016/12/governo-publica-novosdados-de-emissao-de-gases-de-efeitoestufa>. Acesso em: 2 agosto 2017.

${ }^{8}$ Bordonal, R. O.; de Figueiredo, E. B.; La Scala, N. Greenhouse gas balance due to the conversion of sugarcane areas from burned to green harvest, considering other conservationist management practices. Global Change Biology Bioenergy 2012, 4, 846. [CrossRef]

${ }^{9}$ Jiang, C. M.; Yu, W. T.; Ma, Q.; Xu, Y. G.; Zou, H. Alleviating global warming potential by soil carbon sequestration: A multi-level straw incorporation experiment from a maize cropping system in Northeast China. Soil \& Tillage Research 2017, 170, 77. [CrossRef]

${ }^{10}$ Schultz, N.; Lima, E.; Pereira, M. G.; Zonta, E. Adubação nitrogenada e potássica na canasoca colhidas com e sem a queima da palhada. Revista Brasileira de Ciência do Solo 2010, 34, 811. [CrossRef]

${ }^{11}$ Intergovernmental Panel on Climate Change - IPCC. In: Climate Change 2001. The Scientific Basis. Contributions of Working Group I of the Third Assessment Report of the Intergovernmental Panel on Climate Change (eds Houghton, J. T.; Ding, Y.; Griggs, D. J.; Noguer, M.; van der Linden, P. J.; Dai, X.; Maskell, K.; Johnson, C. A.), pp. 239-288. Cambridge University Press, Cambridge. [Link]
${ }^{12}$ Sistema de Estimativas de Emissões de Gases de Efeito Estuda (SEEG): Emissões totais, setor agropecuário no ano de 2015. Disponível

em:<http://plataforma.seeg.eco.br/total emi ssion>. Acesso em: 2 agosto 2017.

${ }^{13}{ }^{13}$ Carvalho, J. L. N.; Avanzi, J. C.; Silva, M. L. N.; de Mello, C. R.; Cerri, C. E. P. Potential of soil carbon sequestration in different biomes of Brazil. Revista Brasileira de Ciência do Solo, 2010, 34, 277. [CrossRef]

${ }^{14}$ Bouwman, A.F. Nitrogen oxides and tropical agriculture. Nature, 1998, 392, 866. [CrossRef]

${ }^{15}$ Signor, D.; Pissioni, L. L. M.; Cerri, C. E. P. Emissões de gases de efeito estufa pela deposição de palha de cana-de-açúcar sobre o solo. Bragantia 2014, 73, 113. [CrossRef]

${ }^{16}$ Smith, K. A.; Conen, F. Impacts of land management on fluxes of trace greenhouse gases. Soil Use and Management 2004, 20, 255. [CrossRef]

${ }^{17}$ Oliveira, B. G.; Carvalho, J. L. N.; Cerri, C. E. P.; Cerri, C. C.; Feigl, B. J. Greenhouse gas emissions from sugarcane vinasse transportation by open channel: a case study in Brazil. Journal of Cleaner Production 2015, 94, 102. [CrossRef]

${ }^{18}$ Siqueira-Neto, M.; Galdos, M. V.; Feigl, B.; Cerri, C. E. P.; Cerri, C. C. Direct $\mathrm{N}_{2} \mathrm{O}$ emission factors for synthetic $\mathrm{N}$-fertilizer and organic residues applied on sugarcane for bioethanol production in Central-southern Brazil. Global Change Biology Bioenergy 2015, 8, 269. [CrossRef]

${ }^{19}$ Oliveira, B. G.; Carvalho, J. L. N.; Cerri, C. E. P.; Cerri, C. C.; Feigl, B. J. Soil greenhousegas fluxes from vinasse application in Brazilian sugarcane areas. Geoderma 2013, 77, 200. [CrossRef]

${ }^{20}$ Carmo, J. B.; Filoso, S.; Zotelli, L. C.; de Sousa Neto, E. R.; Pitombo, L. M.; DuarteNeto, P. J.; Vargas, V. P.; Andrade, C. A.; Gava, G. J. C.; Rossetto, R.; Cantarella, H.; Neto, A. E.; Martinelli, L. A. Infield greenhouse gas emissions from sugarcane soils in Brazil: effects from synthetic and organic fertilizer application and crop trash accumulation. Global Change Biology Bioenergy 2013, 5, 267. [CrossRef] 
${ }^{21}$ Giacomini, S. J.; Jantalia, C. A.; Urquiaga, S. S.; Alves, B. J. R. Emissão de óxido nitroso com a aplicação de dejetos líquidosde suínos em solo sob plantio direto. Pesquisa Agropecuária Brasileira 2006, 41, 1653. [Link] ${ }^{22}$ EMBRAPA - Empresa Brasileira de Pesquisa Agropecuária. Manual de métodos de análise de solo. 2. ed., rev. Atual. Rio de Janeiro, 2012 p., 1997.

${ }^{23}$ Paul, E. A.; Clark, F. E.; Soil microbiology and biochemistry. 2nd ed. California: Academic Press, 340p., 1996.

${ }^{24}$ Rochette, P.; Bochove, E. van; Prévost, D.; Angers,D.A.; Côté, D.; Bertrand, N. Soil carbon and nitrogen dynamicsfollowing application of pig slurry for the $19^{\text {th }}$ consecutive year.2. Nitrous oxide fluxes and mineral nitrogen. Soil Science Society of America Journal 2000, 64, 1396. [CrossRef]

${ }^{25}$ Euclydes, R. F. SAEG: sistema para análises estatísticas egenéticas. Versão 5.0. Viçosa: UFV, 1983.

${ }^{26}$ Jantalia, C. P.; dos Santos, H. P.; Urquiaga, S.; Boddey, R. M.; Alves, B. J. R. Fluxes of nitrous oxide from soil under different crop rotations and tillage systems in the South of Brazil. Nutrient Cycling in Agroecosystems 2008, 82, 161. [CrossRef]

${ }^{27}$ Martins, M. R.; Jantalia, C. P.; Polidoro, J. C.; Batista, J. N.; Alves, B. J.R.; Boddey, R. M.; Urquiaga, $\mathrm{S}$. Nitrous oxide and ammonia emissions from $\mathrm{N}$ fertilization of maize crop under no-till in a Cerrado soil. Soil Tillage Research 2015,151, 75. [CrossRef]

${ }^{28}$ Dobbie, K. E.; Smith, K. A. Nitrous oxide emission factors for agricultural soils in Great Britain: the impact of soil water-filled pore space and other controlling variables. Global Change Biology 2003, 9, 204. [CrossRef]

${ }^{29}$ Vargas, V. P.; Cantarella, H.; Martins, A. A.; Soares, J. R.; do Carmo, J. B.; De Andrade, C. A. Sugarcane Crop Residue Increases $\mathrm{N}_{2} \mathrm{O}$ and $\mathrm{CO}_{2}$ Emissions Under High Soil Moisture Conditions. Sugar Tech 2014, 16, 174. [CrossRef]
${ }^{30}$ Blackmer, A. M.; Bremner, J. M. Potencial of soil as a sink for atmospheric nitrous oxide. Geophysical Research Letters 1976, 3, 739. [CrossRef]

${ }^{31}$ Verchot, L. V.; Davidson, E. A.; Cattânio, J. I. L.; Ackerman, I. L.; Erickson, I. L. E.; Keller, M. Land use change and biogeochemical controls of nitrogen oxide emissions from soils in eastern Amazonia. Global Biogeochemical Cycles 1999, 13, 31. [CrossRef]

${ }^{32}$ Chapuis-Lardy, L.; Wrage, N.; Metay, A.; Chottes, J.; Bernouxs, M. Soils, a sink for $\mathrm{N}_{2} \mathrm{O}$ ? A Review. Global Change Biology 2007, 13, 1. [CrossRef]

${ }^{33}$ Galdos, M. V.; Cerri, C. C.; Cerri, C. E. P. Soil carbon stocks under burned and unburned sugarcane in Brazil. Geoderma 2009, 153, 347. [CrossRef]

${ }^{34}$ Pinheiro, E. F. M.; Lima, E.; Ceddia, M. B.; Urquiaga, S.; Alves, B. J. R.; Boddey, R. M. Impact of pre-harvest burning versus trash conservation on soil carbon and nitrogen stocks on a sugarcane plantation in the Brazilian Atlantic forest region. Plant and Soil 2010, 333, 71. [CrossRef]

${ }^{35}$ Thorburn, P. J.; Meier, E. A.; Collins, K.; Robertson, F. A. Changes in soil carbon sequestration, fractionation and soil fertility in response to sugarcane residue retention are site-specific. Soil and Tillage Research 2012, 120, 99. [CrossRef]

${ }^{36}$ Baggs, E. M.; Rees, R. M.; Smith, K. A.; Vinten, A. J. A. $\mathrm{N}_{2} \mathrm{O}$ emission from soils after incorporating crop residues.Soil Use and Management 2000, 16, 82. [CrossRef]

${ }^{37}$ Duxbury, J. M.; Bouldin, D. R.; Terry, R. E.; Tate, R. L. Emissions of $\mathrm{N}_{2} \mathrm{O}$ from soils. Nature 1982, 298, 462. [CrossRef]

${ }^{38}$ Baggs, E. M.; Stevenson, M.; Pihlatie, A.; Regar, H.; Cook, H.; Cadish, G. $\mathrm{N}_{2} \mathrm{O}$ emissions following application of residues and fertilizer under zero and conventional tillage. Plant and Soil 2003, 254, 361. [CrossRef] 